\title{
BOUND-TO-CONTINUUM TRANSITIONS IN QUANTUM WELL WITH CAPPING BARRIER LAYER OF FINITE SIZE
}

\author{
C. NaLewajko and M. Zalużny
}

Department of Theoretical Physics, Maria Curie-Skłodowska University Pl. M. Curie-Skłodowskiej 1, 20-031 Lublin, Poland

It was shown employing the functional density approach that interference effects resulting from finite size of the cap layer can modify substantially the absorption line shape connected with bound-to-continuum intraband transitions in quantum wells.

PACS numbers: 78.30.Fs, 73.20.Dx

In recent papers $[1,2]$ the problem of quantum well (QW) structures in the vicinity of a high potential with a finite-size capping layer has been discussed. The authors have shown that if a thin cap layer is used, an infinitely high potential which represents the external surface of the semiconductor modifies the energy distribution of carriers around the well. This modification is particularly important for the continuum states because their wave functions are not restricted to the well region. In this paper we present theoretical evidence on the strong influence of the above modification on the absorption line shape connected with bound-to-continuum intraband transitions. The case of infinite-size cap layer (i.e. regular QW) was considered in our previous papers [3, 4].

The absorption of a single quantum well (if we neglect many body corrections) is proportional to $\operatorname{Re} \sigma_{z z}(\omega)$ where $\sigma_{z z}(\omega)$ is the $z z$ component of the complex two-dimensional (2D) conductivity tensor given by [5, 6]

$$
\sigma_{z z}(\omega)=\frac{-\mathrm{i} N_{s} e^{2} \omega}{m} \sum_{f} \frac{f_{1 f}}{\left(\omega_{1 f}^{2}-\omega^{2}\right)-\mathrm{i} \Gamma \omega / \hbar}
$$

where $\Gamma$ is the phenomenological parameter describing the line broadening induced by the electron scattering, $\omega_{1 f}=\left(E_{f}-E_{1}\right) / \hbar, m$ is the effective mass, $f_{1 f}=2 m \omega_{1 f}\left|\left\langle\phi_{f}|z| \phi_{1}\right\rangle\right|^{2} / \hbar$ is the oscillator strength corresponding to $|1\rangle \rightarrow|f\rangle$ transitions and $N_{s}$ is the electron concentration in a unit area. We assume that only ground subband is occupied.

The depolarization effect (DE) and the exciton-like effect (EE) can be included replacing $\sigma_{z z}(\omega)$ by modified 2D conductivity tensor $\widetilde{\sigma}_{z z}(\omega)$ [5]

$$
\tilde{\sigma}_{z z}(\omega)=\frac{-\mathrm{i} N_{s} e^{2} \omega}{m} \sum_{f} \frac{\tilde{f}_{1 f}}{\left(\widetilde{\omega}_{1 f}^{2}-\omega^{2}\right)-\mathrm{i} \Gamma \omega / \hbar}
$$


where

$$
\begin{aligned}
& \left(\hbar \tilde{\omega}_{1 f}\right)^{2}=\tilde{A}_{f f}=\left(\bar{U}^{-1} A \bar{U}\right)_{f f}, \\
& \tilde{f}_{1 f}=\left[\sum_{l}\left(2 m \omega_{1 l} / \hbar\right)^{1 / 2}\left\langle\phi_{l}|z| \phi_{1}\right\rangle \bar{U}_{l f}\right]^{2}
\end{aligned}
$$

and

Here

$$
A_{l f}=\left(\hbar \omega_{1 l}\right)^{2}\left[\delta_{l f}+2 N_{s}\left(\omega_{1 f} / \omega_{1 l}\right)^{1 / 2}\left(1 / \hbar \omega_{1 l}\right)\left(4 \pi e^{2} L_{l f} / \epsilon+B_{l f}\right)\right] .
$$

$$
\begin{aligned}
& L_{l f}=\int_{-\infty}^{\infty} \mathrm{d} z \int_{-\infty}^{z} \mathrm{~d} z^{\prime} \Psi_{1}\left(z^{\prime}\right) \Psi_{l}\left(z^{\prime}\right) \int_{-\infty}^{z} \mathrm{~d} z^{\prime \prime} \Psi_{1}\left(z^{\prime \prime}\right) \Psi_{f}\left(z^{\prime \prime}\right), \\
& B_{l f}=\int_{-\infty}^{\infty} \mathrm{d} z \Psi_{l}(z) \Psi_{f}(z) \Psi_{1}^{2}(z) \frac{\partial v_{\mathrm{xc}}(n(z) ; z)}{\partial n(z)}
\end{aligned}
$$

where $v_{\mathrm{xc}}$ is the exchange-correlation potential, $n(z) \equiv N_{s}\left|\Psi_{1}(z)\right|^{2}$ and $\epsilon$ is the averaged dielectric constant of constituent materials. $\bar{U}$ is the transformation matrix which we define in such a way that $\tilde{A}=\bar{U}^{-1} A \bar{U}$ is diagonal.

We have performed the numerical calculations of the spectral dependence of $\operatorname{Re} \tilde{\sigma}_{z z}(\omega)$ and $\operatorname{Re} \sigma_{z z}(\omega)$ for the resonant GaAs/AlGaAs QW system, i.e. when the second subband $E_{2}$ coincides with the top of the barrier. For simplicity we have neglected: (i) the position and energy dependence of the effective mass, (ii) the influence of the electron-electron interaction on the energy spectrum of the system $[3,4]$. The energy and the wave function of the ground state were obtained assuming that the ground level is localized in the narrow QW of width $a$ and depth $U$. The energies and wave functions of the final (continuum) states are calculated in usual way (see for example Ref. [7]) assuming that these states are confined in a larger box with width $L \gg a$ and infinite potential at the ends. The integrals appearing in the expressions for $\tilde{\sigma}_{z z}(\omega)$ and $\sigma_{z z}(\omega)$ have been calculated numerically taking $L=9000 \AA$. We have checked that increase in $L$ over the above value does not affect the absorption spectrum for the broadening parameter used here. The number of final energy levels in the large box was adjusted so that the absorbance is insensitive to the further increase in the number of the levels incorporated. In our calculations we take the electron effective mass $m=0.066 m_{0}$, the line broadening parameter $\Gamma=7.5 \mathrm{meV}$, dielectric constant $\epsilon=11$ and the electron concentration in a unit area $N_{s}=10^{12} \mathrm{~cm}^{-2}$.

Figure 1 shows the absorption as a function of photon energy for resonant GaAs quantum wells $\left(E_{2}=U\right)$ with different sizes of the cap layer $\left(L_{\mathrm{b}}\right)$ but the same value of separation energy $U-E_{1}$ between the top of the barrier and the ground subband. We find that difference between the line shape for the regular QW $\left[L_{\mathrm{b}}=(L-a) / 2\right]$ and for the semi-infinite QW $\left(L_{\mathrm{b}}=0\right)$ is very small. Much more interesting is the case of the finite size of the cap layer. For $L_{\mathrm{b}}$ in the range 100-1000 $\AA$ we observe very well-pronounced oscillations in the absorption spectrum (connected with the energy-dependent oscillations in the probability of finding the electrons in the cap layer and QW region). It is interesting to note that many body effects (the DE with the EE correction) not only shift the position of the absorption peaks, but also (apart from the main peak) increase their height. 


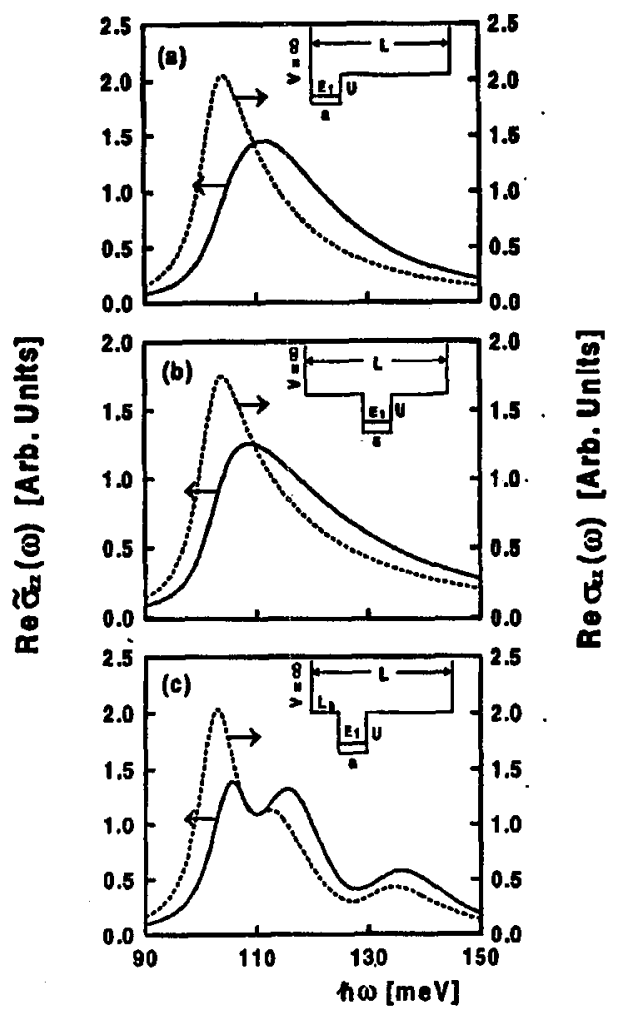

Fig. 1. Absorption characteristics as a function of photon energy $\hbar \omega$ for the resonant GaAs QWs with $U-E_{1}=102 \mathrm{meV}$ and different sizes of the cap layer. (a) Semi-infinite QW with $a=91 \AA$ and $U=145 \mathrm{meV}$; (b) regular QW with $a=60 \AA$ and $U=158 \mathrm{meV}$; (c) QW with $L_{b}=300 \AA, a=61 \AA$ and $U=156 \mathrm{meV}$.

\section{References}

[1] S. Fafard, Phys. Rev. B 46, 4659 (1992).

[2] S. Fafard, E. Fortin, A.P. Roth, Phys. Rev. B 45, 13769 (1992).

[3] M. Załuziny, C. Nalewajko, J. Appl. Phys. 81, 3323 (1997).

[4] M. Zalużny, C. Nalewajko, in: NATO ASI Fabrication, Properties and Application of Low-Dimensional Semiconductor Structures, NATO ASI Series, Ed. M. Balkanski, Kluver, Dordrecht 1995, p. 139.

[5] T. Ando, A. Fowler, F. Stern, Rev. Mod. Phys. 54, 437 (1982).

[6] S.J. Allen, D.C. Tsui, B. Vinter, Solid State Commun. 20, 425 (1976).

[7] Z. Ikonic, V. Milanovic, D. Tjapkin, Appl. Phys. Lett. 54, 247 (1989). 